\title{
Lamina Rara Externa
}

National Cancer Institute

\section{Source}

National Cancer Institute. Lamina Rara Externa. NCI Thesaurus. Code C32919.

One of the three layers comprising the basal lamina of the renal corpuscle membrane that is between the podocytes and the lamina densa. 